\title{
Erythropoietin promotes retinal angiogenesis in a mouse model
}

\author{
CHEN-TING WEN, TAO HE and YI-QIAO XING \\ Eye Center, Renmin Hospital, Wuhan University, Wuhan, Hubei 430060, P.R. China
}

Received November 26, 2013; Accepted June 17, 2014

DOI: $10.3892 / \mathrm{mmr} .2014 .2593$

\begin{abstract}
This study aimed to investigate the effect and potential mechanisms of exogenous administration of recombinant human erythropoietin (rhEPO) on retinal angiogenesis in a mouse model of oxygen-induced retinopathy (OIR). Postnatal day 7 (P7) mice $(n=132)$ were randomly assigned to one of six groups: Control group $(\mathrm{n}=22)$, OIR group $(\mathrm{n}=22), \mathrm{OIR}+$ vehicle control group $(n=22)$, OIR + rhEPO 10 IU group $(n=22)$, OIR + rhEPO 50 IU group $(n=22)$, and OIR + rhEPO $100 \mathrm{IU}$ group $(n=22)$. OIR was induced by exposing mice to $75 \pm 2 \% \mathrm{O}_{2}$ for five days, followed by exposure to room air for a further five days. Animals in groups 3-6 (the OIR + vehicle control group and OIR + rhEPO 10 IU, 50 IU, and 100 IU groups) received an intraperitoneal injection of saline, or rhEPO 10 IU, 50 IU and $100 \mathrm{IU}$, respectively, which were administered daily from P7-P12. Immunofluorescent and hematoxylin-eosin staining were used to detect retinal neovascularization (RNV) in retinal whole mounts. Quantitative polymerase chain reaction and western blot analysis were used to detect the expression levels of vascular endothelial growth factor (VEGF), endothelial nitric oxide synthase (eNOS), and neuronal nitric oxide synthase (nNOS). RNV occurred in the OIR groups and was accompanied by dilated, twisted and occluded blood vessels. rhEPO treatment resulted in an increase in the number of newly formed and severely dilated vessels. rhEPO increased RNV in a dose-dependent manner, which was accompanied by an increase in the messenger RNA and protein expression of VEGF, eNOS and nNOS. Thus, exogenous use of rhEPO promotes the RNV in a mouse model of OIR and is accompanied by increased expression levels of VEGF, eNOS and nNOS.
\end{abstract}

\section{Introduction}

Retinopathy of prematurity (ROP) is a vasoproliferative retinal disease that affects premature babies and is a major cause of

Correspondence to: Mr. Yi-Qiao Xing, Eye Center, Renmin Hospital, Wuhan University, 238 Wuchang Jiefang Road, Wuhan, Hubei 430060, P.R. China

E-mail: xyqdr07@aliyun.com

Key words: oxygen-induced retinopathy, recombinant human erythropoietin, retinal angiogenesis blindness in children (1). ROP has two discrete phases: The first is a phase of delayed vascular growth and the second phase is one of retinal neovascularization (RNV) $(1,2)$. RNV is one of the main contributors to the pathogenesis of ROP (3). Additionally, low birth weight and prematurity are strongly associated with the risk of the disease (2). Previous studies have also revealed that use of recombinant human erythropoietin (rhEPO) is a high risk factor for development of ROP (4-6).

Erythropoietin (EPO) is a glycoprotein that stimulates production of red blood cells, and rhEPO has been used for the treatment of neonatal anemia (7). In addition, rhEPO possesses angiogenic properties, and has been reported to be associated with a high risk of developing ROP (4). In an oxygen-induced retinopathy (OIR) model, suppression of EPO by small interfering RNA inhibited RNV in mice (8), suggesting that EPO promotes neovascularization in OIR. However, the mechanisms by which EPO induces neovascularization remain undetermined. It has been reported that EPO may stimulate postnatal neovascularization in ischemic brain tissues, thereby enhancing blood supply and reducing hypoxia in the area of infarction (9). Therefore, EPO may increase the susceptibility to ROP by increasing RNV.

In the present study, the molecular pathways in ROP were investigated using a mouse model of OIR. The purpose of this study was to examine the effect of exogenous administration of rhEPO on RNV, and to explore possible mechanisms underlying rhEPO-induced neovascularization.

\section{Materials and methods}

Animal model. All experimental protocols were approved by the Institutional Animal Care and Use Committee of Wuhan University (Wuhan, China). Neonate mice (C57BL/6) were obtained from the Animal Center of Wuhan University. All animals $(n=132)$ were housed at room temperature $\left(23 \pm 2^{\circ} \mathrm{C}\right)$. The animals were assigned to six groups. For the control group, animals were housed with room air. For groups 2-6, the OIR groups (OIR, OIR + vehicle control, and OIR + rhEPO 10, 50, and $100 \mathrm{IU})$, litters of postnatal day 7 (P7) pups were housed with their nursing dams in a sealed oxygen chamber, which was controlled to maintain the oxygen concentration at $75 \pm 2 \%$, with a flow rate of $11 / \mathrm{min}$. The oxygen chamber was opened every two days for cleaning and changing of food and water, and every $6 \mathrm{~h}$ for substitution of nursing dams. On postnatal day 12 (P12), the animals were returned to room air until postnatal day 17 (P17). Animals in group 2 were not treated with intraperitoneal (IP) injection of saline or rhEPO. Animals 
Table I. Primer sequences and annealing temperatures of genes.

\begin{tabular}{|c|c|c|c|}
\hline Primer & Sequence (5'-3') & Annealing temperature $\left({ }^{\circ} \mathrm{C}\right)$ & Length (bp) \\
\hline Mouse $\beta$-actin & $\begin{array}{l}\text { F: GTCCCTCACCCTCCCAAAAG } \\
\text { R: GCTGCCTCAACACCTCAACCC }\end{array}$ & 60 & 265 \\
\hline Mouse nNOS & $\begin{array}{l}\text { F: CCTGTGTTCCACCAGGAGAT } \\
\text { R: GTCCCTGGCTAGTGCTTCAG }\end{array}$ & 60 & 249 \\
\hline Mouse eNOS & $\begin{array}{l}\text { F: AGCATACCCCCACTTCTGTG } \\
\text { R: GAAGATATCTCGGGCAGCAG }\end{array}$ & 60 & 208 \\
\hline Mouse VEGF & $\begin{array}{l}\text { F: CAGGCTGCTGTAACGATGAA } \\
\text { R: GCCTTGGCTTGTCACATTTT }\end{array}$ & 60 & 189 \\
\hline
\end{tabular}

nNOS, neuronal nitric oxide synthase; eNOS, endothelial nitric oxide synthase; VEGF, vascular endothelial growth factor.

in groups 3-6 received an IP injection of $0.1 \mathrm{ml}$ saline (group 3 ) or rhEPO at doses of 10, 50 and $100 \mathrm{IU}$ (group 4, 5 and 6, respectively), which were administered daily from $\mathrm{P} 7$ to $\mathrm{P} 12$. On P17, the animals were sacrificed by intravenous injection of pentobarbital $(100 \mathrm{mg} / \mathrm{kg})$ for the following experiments.

Fluorescein retinal angiography assessment of new vessel formation. On P17, four mice from each group were sacrificed, and the eyes were enucleated and fixed in $4 \%$ paraformaldehyde for $1 \mathrm{~h}$ at room temperature. Retinas were isolated and stained overnight at $23^{\circ} \mathrm{C}$ with Isolectin $\mathrm{B}_{4}-594$ Alexa Fluor 594 Conjugate (I21413; Molecular Probes, Eugene, OR, USA; 1:200 dilution) in $1 \mathrm{~mm} \mathrm{CaCl}{ }_{2}$ in phosphate-buffered saline. Retinas were then divided into four equal-sized quadrants and whole mounted. Images of each of the four quadrants of the whole mounted retinas were captured at $\mathrm{x} 4$ magnification using a fluorescence microscope (MP5.0-RTV-CLR-10-C; QImaging, Surrey, BC, Canada). These images were imported and merged together to produce an image of the entire retina for further analysis, using Photoshop CS5 software (Adobe Systems, San Jose, CA, USA). Neovascular tuft formation was measured by comparing the number of pixels in the affected areas with the total number of pixels in the retina. Retinal neovascularization was quantified by measuring the ratio of the neovascular tuft area to the total retinal area. RNV was measured by a researcher blind to the experimental condition.

Hematoxylin-eosin (HE) staining. A total of 6 mice from each group were selected for HE staining. The eyeball was removed, fixed, dehydrated, and embedded in paraffin. Serial sections $(3 \mu \mathrm{m})$ of eyes were cut sagittally at $30 \mu \mathrm{m}$ intervals, and five sections were selected from each eye. The sections were then dried, deparaffinized, rehydrated, and stained with HE. Subsequently the sections were, in turn, dehydrated, cleared, mounted, and viewed under a microscope. The number of endothelial nuclei of newly formed blood vessels that penetrated the inner limiting membrane (ILM) was counted.

Quantitative polymerase chain reaction ( $q P C R$ ). Total RNA was isolated from retinal tissues ( $\mathrm{n}=6$ from each group) using TRIzol (Invitrogen, Carlsbad, CA, USA), according to the manufacturer's instructions. RNA was reverse transcribed into complementary DNA using a First Strand cDNA Synthesis kit (Tiangen Biotech Co., Ltd., Beijing, China). qPCR was performed in a reaction mixture containing $4 \mu \mathrm{l}$ complementary DNA, $0.4 \mu \mathrm{l}$ of each primer $(100 \mu \mathrm{M})$, $10 \mu \mathrm{l}$ SYBR Green/Fluorescein (Thermo Fisher Scientific, Pittsburgh, PA, USA), and 5.2 $\mu \mathrm{l}$ double-distilled $\mathrm{H}_{2} \mathrm{O}$. Primers used for amplification of endothelial nitric oxide synthase (eNOS), neuronal nitric oxide synthase (nNOS), and vascular endothelial growth factor (VEGF) are presented in Table I. $\beta$-actin was used as a housekeeping gene. The reaction conditions were as follows: $50^{\circ} \mathrm{C}$ for $2 \mathrm{~min}, 95^{\circ} \mathrm{C}$ for $10 \mathrm{~min}$, and then 40 cycles of $95^{\circ} \mathrm{C}$ for $30 \mathrm{sec}$ and $60^{\circ} \mathrm{C}$ for $30 \mathrm{sec}$. Melting curve analyses were performed to verify the amplification specificity. The gene expression $\Delta \mathrm{Ct}$ values of messenger RNA (mRNA) of each sample were calculated by normalizing with $\beta$-actin as an internal control. The relative expression levels of eNOS, nNOS and VEGF were calculated using the $2^{-\triangle \Delta C T}$ method.

Western blot analysis. Retinal tissues ( $\mathrm{n}=6$ from each group) were homogenized with cold lysis buffer (on ice). Proteins were isolated by sodium dodecyl sulfate-polyacrylamide gel electrophoresis, and transferred onto polyvinylidene fluoride membranes by electroblotting. Membranes were incubated with the following primary antibodies: Polyclonal rabbit anti-mouse eNOS (dilution 1:300; Beijing Bioss Biotechnology Co., Ltd., Beijing, China), polyclonal rabbit anti-mouse nNOS (dilution 1:300; Beijing Biosynthesis Biotechnology Co. Ltd., Beijing, China) and monoclonal rabbit anti-mouse VEGF (dilution 1:500; Santa Cruz Biotechnology, Inc., Santa Cruz, CA, USA). The membranes were incubated at $4^{\circ} \mathrm{C}$ overnight. Glyceraldehyde-3-phosphate dehydrogenase (GAPDH) was used as loading control. Membranes were then incubated with horseradish peroxidase-linked goat anti-rabbit secondary antibodies (dilution 1:5,000; Wuhan Boster Biological Technology, Ltd., Wuhan, China) at room temperature for $2 \mathrm{~h}$. Bands were visualized using a chemiluminescence detection system (Wuhan Boster Biological Technology, Ltd.), and analyzed using BandScan 5.0 software (Glyko, Novato, CA, USA).

Statistical analyses. Data analyses were performed using SPSS statistical software, version 13.0 J (SPSS, Inc., Chicago, IL, 

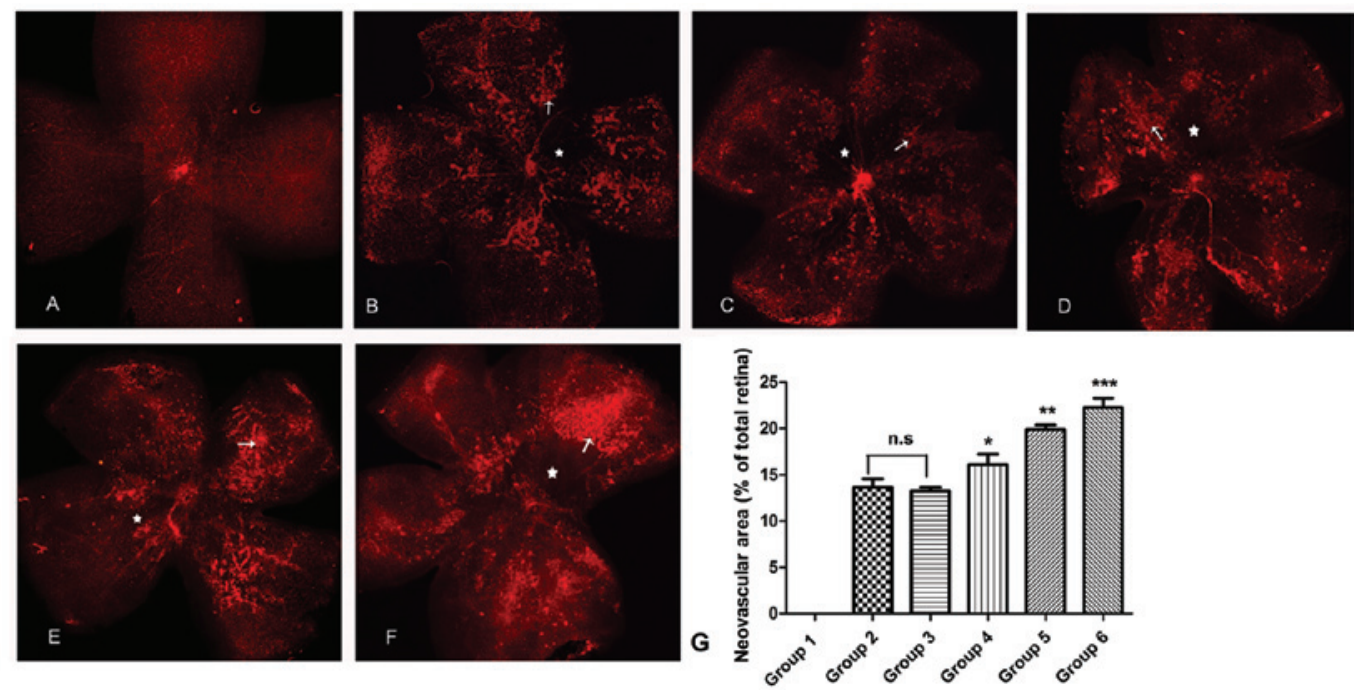

Figure 1. Immunofluorescent images of retinal whole-mounts from P17 mice in (A) control (group 1), (B) oxygen-induced retinopathy (OIR) (group 2), (C) OIR + vehicle control (group 3), (D) OIR + recombinant human erythropoietin (rhEPO) 10 IU (group 4), (E) OIR + rhEPO 50 IU (group 5 ) and (F) OIR + rhEPO $100 \mathrm{IU}$ (group 6) groups. Arrow: Neovascularization. Star: Avascular zone. (G) Neovascular areas in groups 1-6. $\mathrm{n}=8$; ${ }^{*} \mathrm{P}<0.05$ group 3 vs. group $4 ;{ }^{* *} \mathrm{P}<0.05$ group 4 vs. group $5 ;{ }^{* * *} \mathrm{P}<0.05$ group 5 vs. group 6 . Magnification, $\mathrm{x} 4$; staining, Alexa Fluor 594.
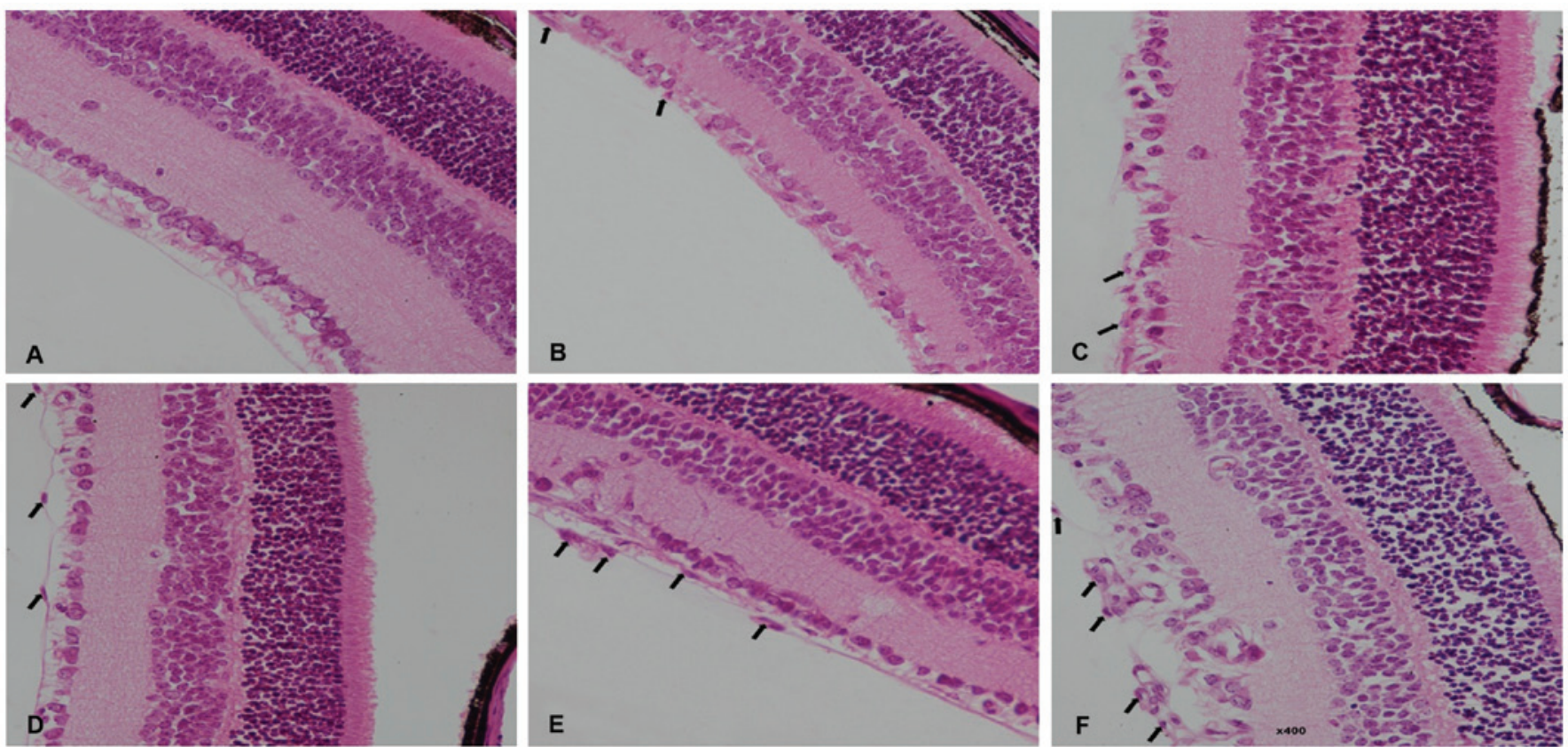

Figure 2. Representative microphotographs of hematoxylin-eosin staining of retinas from P17 mice in (A) control, (B) oxygen-induced retinopathy (OIR), (C) OIR + vehicle control and OIR + recombinant human erythropoietin (D) 10 IU, (E) 50 IU, and (F) 100 IU groups. Newly formed blood vessels are located in the ganglion cell and nerve fiber layers. Arrows: Endothelial cell nuclei penetrating into the inner limiting membrane. Magnification, x40.

USA). All values are presented as the mean \pm standard deviation. Analysis of variance was used to compare differences among the six groups. Fisher's least significant difference t-test was used to compare differences between two groups. $\mathrm{P}<0.05$ was considered to indicate a statistically significant difference.

\section{Results}

rhEPO increases retinal neovascularization. In retinas from control mice, the vessels extended from the optic disc to the periphery, forming a polygonal reticular pattern. Neovascularization and avascular zones were not observed (Fig. 1A). In the retinas from mice in groups $2-6$ (OIR groups), dilated and twisted vessels were observed and certain vessels in the vascular networks were occluded. The normal polygonal reticular pattern and the radial branching pattern were lost in a number of retinal areas and large avascular zones were identified towards the center. Neovascularization was observed between the vascular and avascular zones (Fig. 1B-F). Retinal neovascular areas were measured and compared among groups 1-6. No significant difference in the neovascular area between groups 2 and 3 was identified $(\mathrm{P}>0.05)$. Compared with that of group 3, rhEPO treatment significantly increased the neovascular areas in 


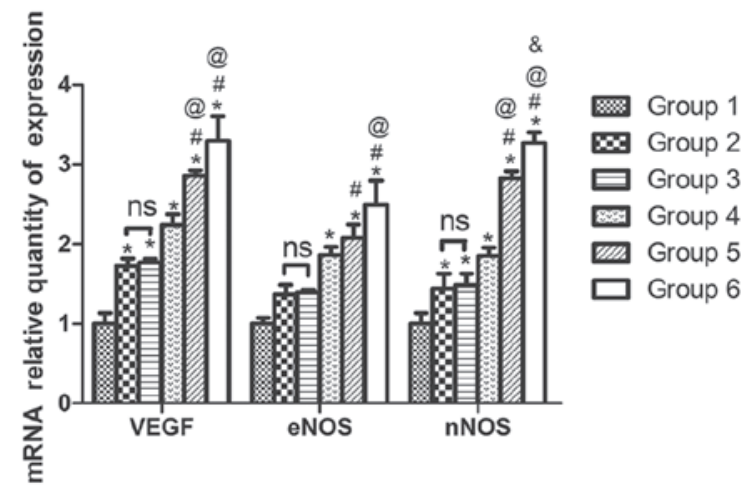

Figure 3. Recombinant human erythropoietin (rhEPO) promotes mRNA expression of endothelial nitric oxide synthase (eNOS), neuronal nitric oxide synthase (nNOS), and vascular endothelial growth factor (VEGF) in an oxygen-induced retinopathy (OIR) mouse model. Quantitative polymerase chain reaction results show the relative expression levels of eNOS, nNOS, and VEGF in retinas from P17 mice in control (group 1), OIR (group 2), OIR + vehicle control (group 3), OIR + rhEPO 10 IU (group 4), OIR + rhEPO $50 \mathrm{IU}$ (group 5), and OIR + rhEPO $100 \mathrm{IU}$ (group 6) groups $\left(\mathrm{n}=6 ;{ }^{*} \mathrm{P}<0.05\right.$ vs. group $1,{ }^{,} \mathrm{P}<0.05$ vs. group $3,{ }^{\circledR} \mathrm{P}<0.05$ vs. group $4,{ }^{\circledR} \mathrm{P}<0.05$ vs. group 5).

groups 4-6 $(\mathrm{P}<0.05)$. rhEPO dose-dependently increased the neovascular areas in group 5 versus group $4(\mathrm{P}<0.05)$ and in group 6 versus group $5(\mathrm{P}<0.05)$ (Fig. 1G).

Quantification of proliferative retinopathy. In paraffin sections of retinas from control mice, no vascular endothelial cells were observed in the vitreous chamber, which is adjacent to the retinal ILM (Fig. 2A). In retinal slices from mice in groups 2-6, cellular nuclei of the vascular endothelium were observed breaking through the retinal ILM (Fig. 2B-F). The degree of neovascularization was quantified by counting the number of endothelial cell nuclei (ECN) which had broken through to the vitreous side of the ILM. In control mice, there was an average of $0.40 \pm 0.70$ nuclei extending into the vitreous chamber, which was significantly lower compared with those of groups $2(35.37 \pm 2.78)(\mathrm{P}<0.001)$ and $3(38.10 \pm 1.90)(\mathrm{P}<0.001)$. No significant difference regarding the number of ECN was identified between groups 2 and $3(\mathrm{P}=0.204)$. Compared with that of group 3, rhEPO treatment increased the number of ECN extending into the vitreous chamber to $42.23 \pm 2.15$ in group 4 $(\mathrm{P}=0.065), 47.56 \pm 3.16$ in group $5(\mathrm{P}=0.001)$, and $55.82 \pm 3.27$ in group $6(\mathrm{P}<0.001)$. In summary, rhEPO dose-dependently increased the number of ECN extending into the vitreous chamber and there were significant differences in the number of ECN extending into the vitreous chamber among groups 4-6 $(\mathrm{P}=0.004)$.

rhEPO increases the MRNA and protein expression of eNOS, nNOS and VEGF in OIR mice. The effects of rhEPO on the mRNA and protein expression levels of eNOS, nNOS and VEGF in OIR mice were examined using qPCR and western blot analysis. qPCR showed that compared with those of group 1, the mRNA levels of nNOS and VEGF were significantly increased in group $2(\mathrm{P}=0.042$ and $\mathrm{P}=0.006$, respectively), but the mRNA levels of eNOS were not significantly increased $(\mathrm{P}=0.124)$. No significant differences in mRNA expression levels of eNOS, nNOS and VEGF were

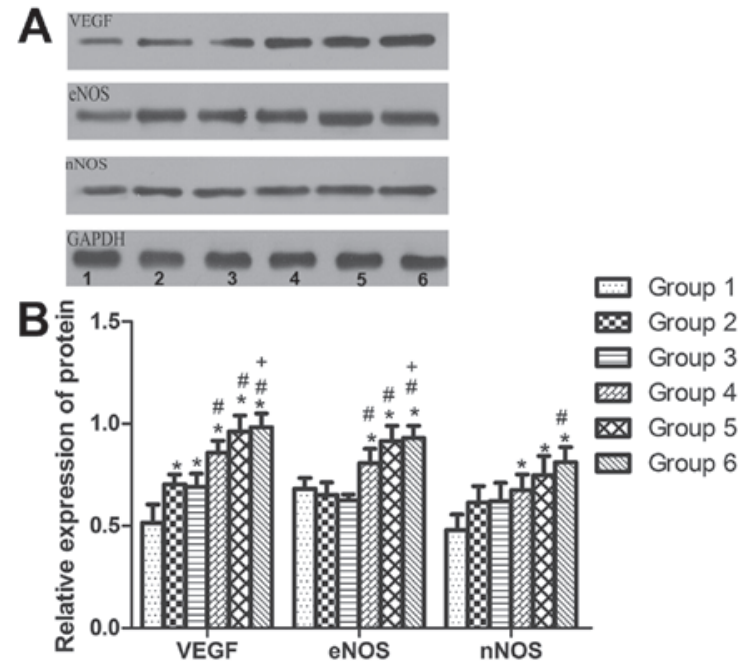

Figure 4. Recombinant human erythropoietin (rhEPO) promotes protein expression of endothelial nitric oxide synthase (eNOS), neuronal nitric oxide synthase (nNOS), and vascular endothelial growth factor (VEGF) in an oxygen-induced retinopathy (OIR) mouse model. (A) Representative western blots showing the expression of eNOS, nNOS, and VEGF in retinas from P17 mice. Lane 1: Control (group 1), lane 2: OIR (group 2), lane 3: OIR + vehicle control (group 3), lane 4: OIR + rhEPO 10 IU (group 4), lane 5: OIR + rhEPO 50 IU (group 5), and lane 6: OIR + rhEPO 100 IU (group 6). (B) Quantitative western blot analysis of the relative expression of eNOS, nNOS, and VEGF of retinas from P17 mice. Glyceraldehyde 3-phosphate dehydrogenase was used as the loading control. ( $\mathrm{n}=6$; ${ }^{*} \mathrm{P}<0.05$ vs. group 1 , ${ }^{\#} \mathrm{P}<0.05$ vs. Group $3,{ }^{+} \mathrm{P}<0.05$ vs. group 4$)$.

identified between groups 2 and $3(\mathrm{P}=0.906, \mathrm{P}=0.840$ and $\mathrm{P}=0.847$, respectively). Compared with those of group 3 , mRNA expression levels of eNOS, nNOS and VEGF were increased in groups 5 and $6(\mathrm{P}=0.010$, and $\mathrm{P}=0.000$ for eNOS, $\mathrm{P}=0.000$ and $\mathrm{P}=0.000$ for $\mathrm{nNOS}$ and $\mathrm{P}=0.000$ and $\mathrm{P}=0.000$ for $\mathrm{VEGF}$, respectively). The upregulation of eNOS, $\mathrm{nNOS}$ and VEGF expression levels by rhEPO was dose-dependent (Fig. 3).

Consistent with the mRNA levels, protein expression levels of eNOS, nNOS, and VEGF were also significantly upregulated in the OIR + rhEPO groups (groups 4-6) compared with those in the control group (group 1) $(\mathrm{P}=0.023, \mathrm{P}=0.000$ and $\mathrm{P}=0.000$ for eNOS, $\mathrm{P}=0.012, \mathrm{P}=0.002$ and $\mathrm{P}=0.000$ for $\mathrm{nNOS}$ and $\mathrm{P}=0.000, \mathrm{P}=0.000$ and $\mathrm{P}=0.000$ for $\mathrm{VEGF}$, respectively). Additionally, protein expression levels of eNOS and VEGF were significantly upregulated in groups 4-6 compared with those in group $3(\mathrm{P}=0.003, \mathrm{P}=0.000$ and $\mathrm{P}=0.000$ for $\mathrm{eNOS}$ and $\mathrm{P}=0.012, \mathrm{P}=0.000$ and $\mathrm{P}=0.000$ for $\mathrm{VEGF})$. Expression levels of nNOS were increased in group 6 compared with those in group $3(\mathrm{P}=0.014)$. rhEPO dose-dependently increased protein expression of eNOS, nNOS and VEGF (Fig. 4).

\section{Discussion}

rhEPO has previously been used in the treatment of anemia, and has subsequently been identified as an effective treatment for neonatal anemia and anemia caused by cancer treatment (7,10-12). Numerous clinical and preclinical studies have shown that use of rhEPO is associated with a high risk of developing ROP (4-6,8), although one small clinical study has reported that such use is not associated with the incidence and severity of ROP (13). 
Certain large scale clinical trials have demonstrated the association of rhEPO with a high risk of developing ROP $(6,14)$. Figueras-Aloy et al $(6)$ reported that EPO plus iron administration was associated with a high risk of grade 1 ROP. Brown et al (14) reported that rhEPO dose-dependently increased the risk of ROP, and that all patients who underwent laser coagulation were treated with rhEPO. However, the mechanisms by which EPO increases the risk of ROP are not well established. In the present study, it was determined that rhEPO treatment promoted RNV in a mouse model of OIR, which was accompanied by an increase in the expression levels of eNOS, nNOS and VEGF. In this respect, the present study suggests that eNOS, nNOS and VEGF may mediate neovascularization induced by rhEPO in ROP.

The development of ROP includes two phases: A first phase of oxygen-induced vessel attenuation and a second phase of hypoxia-induced vasoproliferation $(1,2)$. The initial period of retinal vascular attenuation occurs when an infant undergoes oxygen therapy, and subsequent RNV is promoted by exposure of the infant to room air (15). The newly formed blood vessels are fragile and rupture easily, leading to severe complications such as intravitreous hemorrhage and retinal detachment. In the present study, an ROP mouse model was used, in which P7 mice were first raised in a medium with a high oxygen concentration to mimic the first stage of ROP, and then subsequently returned to room air on P12 to mimic the second stage of ROP. This model has been demonstrated to be a good animal model of human ROP (16).

Once the mouse model of ROP was established, immunofluorescent microscopy was used to investigate the morphology of retinal vascular networks in whole-mounted retinas. It was determined that abnormal neovascularization occurred in the OIR group, which was accompanied by dilated, twisted and occluded blood vessels. rhEPO treatment resulted in an increase in the number of newly formed and severely dilated and occluded vessels. In addition, the degree of neovascularization was quantified by measuring the ratio of the neovascular tuft area to the total retinal area and counting the number of ECN on the vitreous side of the ILM. In normal conditions, there are no neovascularized or avascular zones in flat-mounted retinas, and blood vessels in the vitreous chamber and retinal vessels do not extend out into the ILM. Therefore, neovascular areas and blood vessels that extend into the vitreous chamber across the ILM are regarded as abnormal neovascularization. Counting the number of ECN on the vitreous side of the ILM has been used as the gold standard for studying RNV. In the present study, it was revealed that the number of ECN extending into vitreous chamber was significantly greater in the OIR group compared with that in the control group, suggesting that the OIR model successfully induced RNV. Treatment with rhEPO increased the number of ECN extending into the vitreous chamber in a dose-dependent manner, further suggesting that EPO promotes RNV.

VEGF is one of the most potent proangiogenic factors and it induces endothelial cell proliferation and promotes angiogenesis (17). VEGF induces angiogenesis through direct stimulation of VEGF receptors on vascular cells, leading to increased permeability and neovascularization in vivo. Sato et al (18) reported that the elevated level of EPO in eyes with stage 4 ROP was correlated with the level of VEGF, suggesting that EPO and VEGF contribute to the development of ROP. Furthermore, VEGF has been shown to mediate angiogenesis induced by erythropoiesis-stimulating agents (19). The present study revealed that rhEPO treatment dose-dependently increased RNV in an OIR mouse model, which was accompanied by an increase in levels of VEGF expression. The rhEPO-induced upregulation of VEGF was dose-dependent, suggesting that rhEPO may promote VEGF expression, thus contributing to the pathogenesis of RNV.

NOSs, including constructive and inducible NOS, are a family of enzymes that catalyze the production of nitric oxide from L-arginine. Nitric oxide is an important cellular signaling molecule that is involved in angiogenesis (20). VEGF stimulates the release of nitric oxide from cultured human umbilical venous endothelial cells and upregulates the expression of NOS.

Rezaeian et al (21) reported that EPO promoted angiogenesis and prevented musculocutaneous tissues from ischemic damage by upregulating the expression of eNOS and nNOS. Concurrently, the present study revealed that rhEPO treatment promoted RNV and induced significantly higher levels of expression of eNOS and nNOS in an OIR mouse model. Upregulation of eNOS and nNOS induced by rhEPO was dose-dependent, suggesting that rhEPO may contribute to the development of ROP by inducing the expression of eNOS and nNOS.

In conclusion, this study demonstrated that rhEPO treatment promoted RNV in an OIR mouse model, suggesting that EPO contributes to the pathogenesis of RNV. It was also revealed that rhEPO treatment results in an increase in the mRNA and protein expression levels of VEGF, eNOS and nNOS, suggesting that rhEPO regulates RNV via upregulation of VEGF, eNOS and nNOS. Thus, this study provides a theoretic basis for prevention of rhEPO-induced RNV in a clinical setting.

\section{References}

1. Smith LE: Pathogenesis of retinopathy of prematurity. Growth Horm IGF Res 14 (Suppl A): S140-S144, 2004.

2. Hartnett ME and Penn JS: Mechanisms and management of retinopathy of prematurity. N Engl J Med 368: 1162-1163, 2013.

3. ShresthaJB,BajimayaS,SharmaA,ShresthalJandKarmacharyaP: Incidence of retinopathy of prematurity in a neonatal intensive care unit in Nepal. J Pediatr Ophthalmol Strabismus 47: 297-300, 2010.

4. Romagnoli C, Tesfagabir MG, Giannantonio C and Papacci P: Erythropoietin and retinopathy of prematurity. Early Hum Dev 87 (Suppl 1): S39-S42, 2011.

5. Suk KK, Dunbar JA, Liu A, et al: Human recombinant erythropoietin and the incidence of retinopathy of prematurity: a multiple regression model. J AAPOS 12: 233-238, 2008.

6. Figueras-Aloy J, Alvarez-Domínguez E, Morales-Ballus M, Salvia-Roiges MD and Moretones-Suñol G: Early administration of erythropoietin in the extreme premature, a risk factor for retinopathy of prematurity? An Pediatr (Barc) 73: 327-333, 2010 (In Spanish).

7. Shannon K: Recombinant human erythropoietin in neonatal anemia. Clin Perinatol 22: 627-640, 1995.

8. Xiong SQ, Xia XB, Xu HZ and Jiang J: Suppression of retinal neovascularization by small-interference RNA targeting erythropoietin. Ophthalmologica 223: 306-312, 2009.

9. Heeschen C, Aicher A, Lehmann R, et al: Erythropoietin is a potent physiologic stimulus for endothelial progenitor cell mobilization. Blood 102: 1340-1346, 2003.

10. Zuppa AA, Alighieri G, Fracchiolla A, et al: Comparison between two treatment protocols with recombinant human erythropoietin (rHuEpo) in the treatment of late anemia in neonates with Rh-isoimmunization. Pediatr Med Chir 34: 186-191, 2012. 
11. Thomaidis T, Weinmann A, Sprinzl M, et al; Upper-GI-Group of AIO (Arbeitsgemeinschaft Internistische Onkologie), Germany: Erythropoietin treatment in chemotherapy-induced anemia in previously untreated advanced esophagogastric cancer patients. Int J Clin Oncol 19: 288-296, 2013.

12. Fenner $\mathrm{MH}$ and Ganser A: Erythropoietin in cancer-related anemia. Curr Opin Oncol 20: 685-689, 2008.

13. Shah N, Jadav P, Jean-Baptiste D, et al: The effect of recombinant human erythropoietin on the development of retinopathy of prematurity. Am J Perinatol 27: 67-71, 2010.

14. Brown MS, Barón AE, France EK and Hamman RF: Association between higher cumulative doses of recombinant erythropoietin and risk for retinopathy of prematurity. J AAPOS 10: 143-149, 2006.

15. Patz A: Current concepts of the effect of oxygen on the developing retina. Curr Eye Res 3: 159-163, 1984.

16. Smith LE, Wesolowski E, McLellan A, et al: Oxygen-induced retinopathy in the mouse. Invest Ophthalmol Vis Sci 35: 101-111, 1994.
17. Neufeld G, Cohen T, Gengrinovitch S and Poltorak Z: Vascular endothelial grow th factor (VEGF) and its receptors. FASEB J 13: 9-22, 1999.

18. Sato T, Kusaka S, Shimojo H and Fujikado T: Vitreous levels of erythropoietin and vascular endothelial growth factor in eyes with retinopathy of prematurity. Ophthalmology 116: 1599-1603, 2009.

19. McVicar CM, Colhoun LM, Abrahams JL, et al: Differential modulation of angiogenesis by erythropoiesis-stimulating agents in a mouse model of ischaemic retinopathy. PLoS One 5: e11870, 2010.

20. Cooke JP and Losordo DW: Nitric oxide and angiogenesis. Circulation 105: 2133-2135, 2002.

21. Rezaeian F, Wettstein R, Egger JF, et al: Erythropoietininduced upregulation of endothelial nitric oxide synthase but not vascular endothelial growth factor prevents musculocutaneous tissue from ischemic damage. Lab Invest 90: 40-51, 2010 . 